\title{
Anaemia and Associated Factors Among Pregnant Women Attending Antenatal Care Follow-Up at Yabello General Hospital Living in Pastoralist Borena Zone, Oromia, Ethiopia From From July to August, 2019
}

Godana Arero ( $\square$ godanag769@gmail.com )

Adama Science and Technology University

Kinde Asssefa

Yaba College of Technology

\section{Research}

Keywords: Magnitude, Anemia, Antenatal Care, Yabello Hospital, Borena, Ethiopia

Posted Date: June 15th, 2020

DOI: https://doi.org/10.21203/rs.3.rs-33531/v1

License: (c) (1) This work is licensed under a Creative Commons Attribution 4.0 International License. Read Full License 


\section{Abstract}

Objective: The purpose of the study is to assess magnitude of anemia and associated factors among pregnant women attending antennal care follow-up at Yabello General Hospital in Pastoralist Borena Zone from July-August, 2019.

Method: A hospital based cross-sectional study design was employed among 265 pregnant women attending antenatal care at Yabello General Hospital from June 17-August 16 2019. A systematic random sampling technique was used to select two hundreds sixty five study subjects. The first study subject was chosen randomly by simple random sampling method blindly picking one of two using pieces of papers named for the first two visitors. The sampling interval (K) calculated to be 2 , and then, every second pregnant woman who attending antenatal care was recruited.Socio-demographic, maternal nutrition, information and obstetric and medical characteristics were assessed. Hemoglobin value, stool examination, HIV and syphilis test results were collected from their regular laboratory tests. Blood film was conducted for pregnant women who had signs and symptoms and whose hemoglobin value less than the established cut of values and data were analyzed using SPSS version 20.0 software

Results: Magnitude of anemia with median hemoglobin value were $(11.10 \mathrm{~g} / \mathrm{dl} \pm 1.66)$; majority 46(63.9\%) had mildly anemia, 24(33.3\%) moderate and 2(2.8\%) were severe anemia. Urban dwellers women (AOR, 95\% Cl: .18(.05-.64)), for those who had abortion before current pregnancy (AOR, 95\% Cl: 3.08(1.17-8.13)); coffee/tea drinking immediately after meal (AOR, 95\% Cl: 4.39(1.82-10.59), \& who had excessive menstrual bleeding before current pregnancy were (AOR, 95\% Cl: 3.39(1.47-7.84)) \& mid-upper arm circumference less than $23 \mathrm{~cm}$ (AOR, 95\% Cl: 6.27(1.15-14.30)) were found to be independent predictors of anemia among pregnant women.

Conclusion: Anemia in study area among pregnant women in Ethiopia was higher as compare with similar study elsewhere. Malnutrition, abortion, excessive bleeding \& nutrition interaction with other inhibitors like coca cola, tea and coffee immediately after meals were independent predictors for anemia.

\section{Background:}

Anemia is defined as a condition in which the number and size of red blood cells and/or the concentration of hemoglobin falls below an established normal cut-off value, and as a result it impaired oxygen-carrying capacity of the red blood cells to the tissues and carbon dioxide from the tissues to the lung (1). Anemia during pregnancy is a global public health problem which affecting the population of both developed and developing countries with various type and magnitudes and which is responsible for the occurrences of major negative consequences on the human health, the social and the economic development of the countries (2). During pregnancy, the concentration of hemoglobin decreases from the normal with hemo-dilution due to the increasing of the plasma volume in the circulating blood, and this condition was increase the needs of iron and other nutrients by the body three to six times more than the normal condition for both the mothers and fetus (3).The causes for the occurrences of most anemia 
during pregnancy include; pregnancies that are closed together, being illiterate, had anemia before the current pregnancy, no eating of enough foods that are rich with iron, poor absorption of iron by the body, parasitic infections, parity, gravidity, poverty, culture, infections, less awareness about anemia, excessive blood loss during pregnancy poor health seeking behavior and so on $(6,8)$. The occurrence of anemia during pregnancy is preventable and reversible when it occurs due to deficiencies of nutrients particularly, micronutrients, vitamins and proteins and adverse effects of smoking on vitamin C, vitamin B12, folic acid and vitamin A, and therefore, according to Centers of Disease Control and Prevention (CDC) and World Health Organization (WHO), anemia during pregnancy is defined as the concentration of hgb. less than $11 \mathrm{~g} / \mathrm{dl}(7,9)$. The magnitudes of anemia among pregnant women vary from countries to countries due to socially, geographically and economic status of the countries, and hence, in Sub-Saharan Africa countries the magnitude of anemia among pregnant women is $57.1 \%$, in south-east Asia $48.2 \%$, and this magnitude is twice as common as in America $21.1 \%$ and Europe $25.1 \%$ (10).

2. Figure 1. The Conceptual Frame work adapted from Abie A. 2018

\section{Methods:}

The study aimed to assess magnitude of anemia and associated factors among pregnant women attending antennal care follow-up at Yabello General Hospital in Pastoralist Borena Zone, Ethiopia from July-August, 2019. A hospital based cross-sectional study design was applied.

The source population for this study was all pregnant women with the age between 15 to 49 years old who live in Yabello Town and its catchment area. The study population was pregnant women who attending antenatal care in Yabello General Hospital and study subjects were pregnant women who give full information for research team. Inclusion criteria was a pregnant women who living in the catchment area and able to respond to the interview whereas exclusion criteria was a pregnant woman who severely ill and unable to respond to the interview and those who referred from other health facilities.

Sampling technique: A systematic and simple random sampling technique was used. The first study subject was chosen simple random sample by blindly picking one of two using pieces of papers named for the first two visitors. The sampling interval $(\mathrm{K})$ calculated to be 2 , and then, every second pregnant woman who attending antenatal care was recruited.

Data collection procedure: Data was collected using a face to face interview and MUAC measurement by trained duty-off mid-wife nurses at the ANC clinic and the results of stool 
investigation, the value of hgb. and PHICT (HIV) were collected by duty-off assigned laboratory technologists in the laboratory and recorded on to the corresponding questionnaires immediately after the final results were approved by the laboratory technologists. A blood smear was conducted to investigate the morphology of RBCs for those whose hgb. value was less than the established cut-off value $(<11.0 \mathrm{~g} / \mathrm{dl})$.

Statistical analysis: Data was entered in to Epi- Info version 7.0 and then exported to SPSS Software Version 20.0. Descriptive statistics were done and summarized by frequencies proportions for categorical predictors. Variables with $p<0.25$ in a binary logistic regression were subjected to a multivariate logistic regression to control the possible confounding effects of other predictors and the strength of association was estimated using odds ratio with $\mathrm{p}<0.05$ at $95 \%$ confidence interval.

Data quality management: The questionnaire is adapted from prior related studies and modified to the context of the study area and was translated in to local languages by whom, who is familiar with both languages and back to English. A pre-test was done on $5 \%$ with similar population of non-pregnant women in outpatient department at Yabello general hospital to check the understandability of the questionnaire. Data collectors were oriented on data collection tools and the procedures. In the laboratory, the quality of results was ensured through appropriate samples collection and analysis by experienced laboratory personnel. Samples were analyzed using internationally recommended HemoCue-301 hgb. analyzer and Olympus microscope. Appropriate standard operating procedures during sample collection and investigations were followed and completeness of data was checked by supervisor and principal investigator in a daily basis. The value of hgb. was adjusted for altitude and trimesters using WHO hgb. adjustment factors (44).

\section{Sample size determination:}

The sample size was determined using both single population proportion formula by computing the proportion of anemia among pregnant women from Arba Minch was 32.8 $\%$ (46), 5\% of margin of error and 95\% confidence interval and a double population proportion formula using Stat-calc Epi Info statistical software version 7.0 with the following assumptions: Confidence level $=95 \%$, Power $=80 \%$

\section{Sample size determination for the first objective:}

Assumption:

$$
\mathrm{n}=\underline{\mathrm{Z} / \llbracket} \underline{\underline{2}} \underline{\mathrm{P}}(\underline{1-\mathrm{P}}) .
$$


(d) ${ }^{2}$

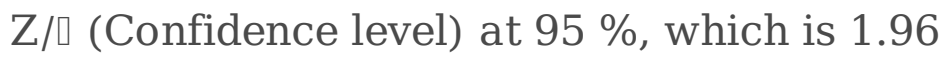

$\mathrm{P}$ is the proportion of anemia among pregnant women which is assumed to be $32.8 \%$

(Bekele, et al.)

d (margin of error) which is $5 \% 185,980887$

$$
\mathrm{n}=(\underline{1.96})^{2} \stackrel{2}{*} \frac{0.33 *}{(0.05)^{2}}(\underline{1-0.33}) .=340
$$

Since the total population is less than 10,000 , the final sample size needs to be corrected using the correction formula:

$$
\begin{array}{r}
\mathrm{n}_{\mathrm{f}}=\mathrm{n} * \mathrm{~N} \\
\mathrm{n}+\mathrm{N}
\end{array}
$$

Where; $n_{f}=$ the desired sample size (when the study population is less than $10,000)$

$\mathrm{n}=$ the desired sample size (when the study population is greater than 10,000 )

$\mathrm{N}=$ the average pregnant women who is expected to visit the ANC during the study period of two months is 547 (YGH, HMIS March to April report 2019).

$$
\mathrm{n}_{\mathrm{f}}=\frac{340 * 547}{340+547}=210
$$

The final sample size including $10 \%$ non-response rate is calculated to be 231

\section{Sample size determination for second objectives:}

The sample size for the second objective was calculated using double population proportion formula using the Stat-calc of Epi Info statistical software version 7.0 with the following assumptions: Confidence level $=95 \%$, Power $=80 \%$, the ratio of unexposed to exposed 1.5.

Table 1: Sample size calculation from prior related studies for some selected factors associated with anemia among pregnant women attending ANC at Yabello general hospital from July to August 2019 


\begin{tabular}{|c|c|c|c|c|c|}
\hline \multirow[t]{2}{*}{ Variables } & \multirow[t]{2}{*}{$\mathrm{AOR}$} & \multicolumn{2}{|c|}{ Anemia } & \multirow{2}{*}{$\begin{array}{l}\text { Sample } \\
\text { size }\end{array}$} & \multirow[b]{2}{*}{ References } \\
\hline & & Exposed & $\begin{array}{l}\text { Non- } \\
\text { exposed }\end{array}$ & & \\
\hline HIV infection & $\begin{array}{l}2.72(1.04- \\
7.23)\end{array}$ & $8 \%$ & $42 \%$ & 120 & $\begin{array}{l}\text { (Melaku et } \\
\text { al., 2014) }\end{array}$ \\
\hline $\begin{array}{l}\text { Previous malaria } \\
\text { attack }\end{array}$ & $\begin{array}{l}1.70(1.40- \\
9.33)\end{array}$ & $58.7 \%$ & $47.0 \%$ & $\underline{260}$ & $\begin{array}{l}\text { (Mihiret et } \\
\text { al., 2015) }\end{array}$ \\
\hline $\begin{array}{l}\text { No iron } \\
\text { supplementation }\end{array}$ & $\begin{array}{l}1.96(0.85- \\
4.45)\end{array}$ & $36.7 \%$ & $63.3 \%$ & 148 & $\begin{array}{l}\text { (Ayano et } \\
\text { al., 2018) }\end{array}$ \\
\hline $\begin{array}{l}\text { No information } \\
\text { about anemia }\end{array}$ & $\begin{array}{l}2.16(0.9- \\
5.16)\end{array}$ & $31.7 \%$ & $68.3 \%$ & 136 & $\begin{array}{l}\text { (Zekarias } \\
\text { et al., } \\
2017 \text { ) }\end{array}$ \\
\hline
\end{tabular}

The maximum sample size that was obtained from the second objective is 260; therefore, this sample is the larger sample size than the sample size from the first objective which was 231, therefore the final sample size that used for this study was the sample size that obtained from the second objective (260).

\section{Results:}

A total 265 pregnant women were participated in the study with $100 \%$ response rate. The mean age was $(23.77 \pm 4.91)$. Look table 1 for more detail)

Table1. Anemia and socio-demographic characteristics of study participants 


\begin{tabular}{|c|c|c|c|c|}
\hline \multirow[t]{2}{*}{ Variables } & \multirow[t]{2}{*}{ Categories } & \multicolumn{2}{|r|}{ Anemic } & \multirow[t]{2}{*}{ Total (\%) } \\
\hline & & Yes (\%) & No $(\%)$ & \\
\hline \multirow[t]{6}{*}{ Age } & $15-19$ & $19(23.2)$ & $63(76.8)$ & $82(30.9)$ \\
\hline & $20-24$ & $29(26)$ & $81(73.6)$ & $110(41.5)$ \\
\hline & $25-29$ & $17(67.3)$ & $35(32.7)$ & $52(19.6)$ \\
\hline & $30-34$ & $4(28.6)$ & $10(71.4)$ & $14(5.3)$ \\
\hline & $35-39$ & $2(33.3)$ & $4(66.7)$ & $6(2.3)$ \\
\hline & $40-44$ & $1(100)$ & 0 & $1(0.4)$ \\
\hline \multirow[t]{2}{*}{ Address } & Urban & $25(15.4)$ & $137(84.6)$ & $162(61.1)$ \\
\hline & Rural & $47(44.6)$ & $56(54.4)$ & $103(28.9)$ \\
\hline \multirow[t]{4}{*}{ Religion } & Protestant & $28(25.5)$ & $82(74.5)$ & $110(41.5)$ \\
\hline & Muslim & 11(17.7) & $51(82.3)$ & $62(23.4)$ \\
\hline & Orthodox & $7(14.9)$ & $40(85.1)$ & $47(17.7)$ \\
\hline & Waqefeta & $26(56.5)$ & $20(43.5)$ & $46(17.4)$ \\
\hline \multirow{4}{*}{ Educational level } & Illiterate & $43(41.3)$ & $61(58.7)$ & $104(39.2)$ \\
\hline & Read \& write & $7(36.8)$ & $12(63.2)$ & $19(7.2)$ \\
\hline & Primary/high school & $14(15.9)$ & $74(84.1)$ & $88(33.2)$ \\
\hline & College & $8(14.8)$ & $46(85.2)$ & $54(20.4)$ \\
\hline \multirow[t]{2}{*}{ Marital status } & Married & $70(27.1)$ & 188(72.9) & $258(97.4)$ \\
\hline & Not married & $2(28.6)$ & $5(71.4)$ & $7(2.6)$ \\
\hline \multirow[t]{4}{*}{ Family size } & $1-2$ & $3(37.5)$ & $5(62.5)$ & $8(1.1)$ \\
\hline & $3-4$ & $22(22.4)$ & 76(77.6) & $98(37.0)$ \\
\hline & $5-6$ & $15(22.4)$ & $52(77.6)$ & $67(25.3)$ \\
\hline & $6 \&$ above & $32(34.8)$ & $60(65.2)$ & $92(35.7)$ \\
\hline \multirow[t]{3}{*}{ Monthly average income } & $<1000$ EB & $40(44.4)$ & $50(55.6)$ & $90(33.9)$ \\
\hline & 1001-2000EB & $6(25.0)$ & $18(75.0)$ & $24(9.1)$ \\
\hline & $>2000 \mathrm{~EB}$ & $26(17.2)$ & $125(82.8)$ & $151(57.0)$ \\
\hline \multirow[t]{4}{*}{ Occupation } & House wives & $49(31.4)$ & 107(68.6) & $156(58.9)$ \\
\hline & Daily laborer & $1(50.0)$ & $1(50.0)$ & $2(0.8)$ \\
\hline & Gov. employee & $7(13.2)$ & $46(86.8)$ & $53(20.0)$ \\
\hline & Merchant & $15(27.8)$ & $39(72.2)$ & $54(20.4)$ \\
\hline
\end{tabular}

\section{Magnitude and severity of anemia}

Overall magnitude of anemia among the studied participants was $27.2 \%$ with the media hemoglobin range $(11.10 \mathrm{~g} / \mathrm{dl} \pm 1.66)$. Regarding the morphologic appearance of the red blood cells 25(9.4\%) pregnant women were with micro-cytosis (smaller size than the normal red blood cells size).

Anemia and Nutrition Status: One hundred sixty six (62.6\%) participants had meal frequency 3 \& + per day during study period. Among them $32(19.3 \%)$ were anemic, $157(59.2 \%)$ who used to drink coffee/tea immediately after meal had mild anemia, 40(25.5\%) were moderate anemia, and 24(15.3\%) were severe anemia. 
Table2. Anemia and Maternal Nutrition Status

\begin{tabular}{|c|c|c|c|c|}
\hline \multirow[t]{2}{*}{ Variables } & \multirow[t]{2}{*}{ Categories } & \multicolumn{2}{|c|}{ Anemic } & \multirow[t]{2}{*}{ Total (\%) } \\
\hline & & Yes (\%) & No (\%) & \\
\hline \multirow[t]{3}{*}{ Meal freq./ day } & Once per day & $9(36.0)$ & $16(64.0)$ & $25(9.5)$ \\
\hline & Twice per day & $31(41.9)$ & $43(58.1)$ & $74(27.9)$ \\
\hline & $\begin{array}{l}\text { Three times/ } \\
\text { day }\end{array}$ & $32(19.3)$ & $134(80.7)$ & $166(62.6)$ \\
\hline \multirow{2}{*}{$\begin{array}{l}\text { Coffee/tea drinking immediately after } \\
\text { meal }\end{array}$} & Yes & $61(38.9)$ & $96(61.1)$ & $157(59.2)$ \\
\hline & No & $11(10.2)$ & $97(89.8)$ & $108(40.8)$ \\
\hline \multirow{4}{*}{$\begin{array}{l}\text { Green leafy vegetable consuming } \\
\text { status }\end{array}$} & Every day & $3(13.0)$ & $20(87.0)$ & $23(8.7)$ \\
\hline & Once per week & $33(22.8)$ & $112(77.2)$ & $145(54.7)$ \\
\hline & $\begin{array}{l}\text { Once per } \\
\text { month }\end{array}$ & $22(30.6)$ & $50(69.4)$ & $72(27.2)$ \\
\hline & Not at all & $14(66.0)$ & $11(44.0)$ & $25(9.4)$ \\
\hline \multirow[t]{4}{*}{ Fruit consuming status } & Every day & $1(0.4)$ & $2(0.8)$ & $3(1.1)$ \\
\hline & Once per week & $\begin{array}{l}28 \\
(10.6)\end{array}$ & $92(34.7)$ & $120(35.3)$ \\
\hline & $\begin{array}{l}\text { Once } \\
\text { month }\end{array}$ & $18(7.0)$ & $67(25.3)$ & $85(32.3)$ \\
\hline & Not at all & $25(9.4)$ & $32(12.1)$ & $57(21.5)$ \\
\hline \multirow[t]{4}{*}{ Meat eating habit } & Every day & 0 & $4(100.0)$ & $4(1.5)$ \\
\hline & Once per week & $39(25.2)$ & $116(74.8)$ & $155(58.5)$ \\
\hline & $\begin{array}{l}\text { Once } \\
\text { month }\end{array}$ & $29(29.6)$ & $69(70.4)$ & $98(37.0)$ \\
\hline & Not at all & $4(50.0)$ & $4(50.0)$ & $8(3.0)$ \\
\hline
\end{tabular}

Table 5 Distribution of anemia with maternal information characteristics of pregnant women attending antenatal care at Yabello general hospital 17 June-16 August 2019 


\begin{tabular}{|c|c|c|c|c|}
\hline \multirow[t]{2}{*}{ Variables } & \multirow[t]{2}{*}{ Categories } & \multicolumn{2}{|c|}{ Anemic } & \multirow[t]{2}{*}{ Total (\%) } \\
\hline & & Yes (\%) & No $(\%)$ & \\
\hline \multirow[t]{4}{*}{ Information about anemia } & Yes & 27 & $144(84.2)$ & 171(64.5) \\
\hline & & $(15.8)$ & & \\
\hline & No & 45 & $49(52.2)$ & $94(35.5)$ \\
\hline & & $(47.9)$ & & \\
\hline \multirow[t]{2}{*}{ IFAS } & Yes & $42(31.1)$ & $93(68.9)$ & $135(50.9)$ \\
\hline & No & $30(23.1)$ & $100(94.7)$ & $130((49.1)$ \\
\hline \multirow[t]{2}{*}{ Importance of IFAS } & Yes & $23(25.6)$ & $67(74.4)$ & $90(34.4)$ \\
\hline & No & $49(28.0)$ & $126(72.0)$ & $175(66.0)$ \\
\hline \multirow{2}{*}{$\begin{array}{l}\text { Problem associated with anemia during } \\
\text { pregnancy }\end{array}$} & Yes & $31(24.2)$ & $97(75.8)$ & $128(48.3)$ \\
\hline & No & $41(29.9)$ & $96(70.1)$ & 137(51.7) \\
\hline
\end{tabular}

Percentage of anemia by severity among anemic pregnant women $(n=72)$

Red blood cells morphologic distribution among anemic pregnant women $(n=72)$

\section{Obstetric and medical characteristics:}

One hundred four (39.2\%) participants had no previous information about the importance of Iron and Folic Acid Supplementation (IFA), almost 135(50.9\%) respondents were supplemented with IFA during their ANC visits. One hundred five (39.6\%) participants were not using any methods of contraceptive before the occurrence of current pregnancy. Two hundred seventeen (81.8\%) had abortion before the occurrence of the current pregnancy. Two hundred five $(77.4 \%)$ had no malaria history prior to one year of the study period. Nine (3.3\%) participants who had infected with intestinal parasite (amoeba) of this, 7 (2.6\%) of the were anemic and $4(1.5 \%)$ and $3(1.13 \%)$ respondents were mild and moderate anemic respectively (Table 3 ).

Table3. Anemia, Obstetric and Medical characteristics Study participants 


\begin{tabular}{|c|c|c|c|c|}
\hline \multirow[t]{2}{*}{ Variables } & \multirow[t]{2}{*}{ Categories } & \multicolumn{2}{|c|}{ Anemic } & \multirow[t]{2}{*}{ Total (\%) } \\
\hline & & Yes (\%) & No $(\%)$ & \\
\hline \multirow[t]{2}{*}{ IFAS during ANC visits } & Yes & 42 & $93(68.9)$ & 135(50.9) \\
\hline & No & $\begin{array}{l}30 \\
(23.1)\end{array}$ & $100(76.9)$ & $130(49.1)$ \\
\hline \multirow[t]{2}{*}{ Gravidity } & $\begin{array}{l}\text { Prim- } \\
\text { gravidity }\end{array}$ & $\begin{array}{l}28 \\
(24.6)\end{array}$ & $86(75.4)$ & 114(43.0) \\
\hline & $\begin{array}{l}\text { Multi- } \\
\text { gravidity }\end{array}$ & $\begin{array}{l}44 \\
(29.1)\end{array}$ & $\begin{array}{l}107 \\
(70.9)\end{array}$ & $151(57.0)$ \\
\hline \multirow[t]{2}{*}{ OC usage before current pregnancy } & Yes & $17(17.0)$ & $90(84.1)$ & $107(40.4)$ \\
\hline & No & $55(33.3)$ & $\begin{array}{l}103 \\
(65.2)\end{array}$ & 158(59.6) \\
\hline \multirow[t]{2}{*}{ History of malaria } & Yes & $\begin{array}{l}13 \\
(28.3)\end{array}$ & $43(71.7)$ & $60(22.6)$ \\
\hline & No & $\begin{array}{l}55 \\
(26.8)\end{array}$ & $\begin{array}{l}150 \\
(73.2)\end{array}$ & $205(77.4)$ \\
\hline \multirow[t]{2}{*}{ abortion before current pregnancy } & Yes & $23(51.1)$ & $22(48.9)$ & $45(17.0)$ \\
\hline & No & $49(22.3)$ & 171(77.7) & $220(83.0)$ \\
\hline \multirow{2}{*}{$\begin{array}{l}\text { Heavy menstrual bleeding before } \\
\text { current pregnancy }\end{array}$} & Yes & $32(50.8)$ & $31(49.2)$ & $63(23.8)$ \\
\hline & No & $\begin{array}{l}40 \\
(19.8)\end{array}$ & $\begin{array}{l}162 \\
(80.2)\end{array}$ & $202(76.2)$ \\
\hline \multirow[t]{3}{*}{ Gestational age } & $\begin{array}{l}\text { First } \\
\text { trimester }\end{array}$ & $7(30.4)$ & $16(69.6)$ & $23(8.7)$ \\
\hline & $\begin{array}{l}\text { Second } \\
\text { trimester }\end{array}$ & $\begin{array}{l}31 \\
(20.8)\end{array}$ & $\begin{array}{l}118 \\
(79.2)\end{array}$ & $149(56.2)$ \\
\hline & $\begin{array}{l}\text { Third } \\
\text { trimester }\end{array}$ & $\begin{array}{l}34 \\
(35.6)\end{array}$ & $59(63.4)$ & $93(35.1)$ \\
\hline \multirow[t]{3}{*}{ No of ANC visit } & Once & $\begin{array}{l}24 \\
(20.0)\end{array}$ & $96(80.0)$ & $120(45.3)$ \\
\hline & Twice & $\begin{array}{l}29 \\
(35.6)\end{array}$ & $60(64.4)$ & $89(33.6)$ \\
\hline & Three times & $\begin{array}{l}19 \\
(33.9)\end{array}$ & 37 (66.1) & $56(35.1)$ \\
\hline \multirow[t]{3}{*}{ MUAC } & $<21 \mathrm{~cm}$ & $\begin{array}{l}10 \\
(52.6)\end{array}$ & 9 (47.4) & $19(7.2)$ \\
\hline & $21-23 \mathrm{~cm}$ & $\begin{array}{l}55 \\
(27.1)\end{array}$ & $\begin{array}{l}148 \\
(72.9)\end{array}$ & $203(76.6)$ \\
\hline & $>21 \mathrm{~cm}$ & 7 (16.3) & 36 (83.7) & $43(16.2)$ \\
\hline \multirow[t]{2}{*}{ Syphilis } & Positive & $2(25)$ & $6(75)$ & $8(3.0)$ \\
\hline & Negative & $\begin{array}{l}70 \\
(27.2)\end{array}$ & $\begin{array}{l}187 \\
(72.8)\end{array}$ & $257(97.0)$ \\
\hline \multirow[t]{2}{*}{ HIV } & Positive & $2(50.0)$ & $2(50.0)$ & $4(1.5)$ \\
\hline & Negative & $\begin{array}{l}70 \\
(26.8)\end{array}$ & $\begin{array}{l}191 \\
(73.2)\end{array}$ & 261 \\
\hline \multirow[t]{3}{*}{ Stool exam. } & Bacillary & $5(62.5)$ & $3(37.5)$ & $8(3.0)$ \\
\hline & Amoebiasis & $7(77.8)$ & $2(22.2)$ & $9(3.4)$ \\
\hline & No parasite & $\begin{array}{l}65 \\
(24.5)\end{array}$ & $\begin{array}{l}183 \\
(75.5)\end{array}$ & $\begin{array}{l}248 \\
(93.6)\end{array}$ \\
\hline
\end{tabular}




\section{Associated Factors \& Anemia among pregnant women:}

In a binary logistic regression analysis: education, income, abortion, heavy menstrual bleeding, gestational age, family size, meal frequency per day, drinking of coffee immediately after meal and no supplementation of iron-folic acid during pregnancy were statistically significant associate with anemia during pregnancy $(p<0.25)$, (Tables 4-6).

Table 4. Binary Logistic Regression Analysis for Socio-Demographic factors associated with anemia

\begin{tabular}{|c|c|c|c|c|c|}
\hline \multirow[t]{2}{*}{ Variables } & \multirow[t]{2}{*}{ Categories } & \multicolumn{2}{|c|}{ Anemic } & \multirow[t]{2}{*}{ COR $(95 \% \mathrm{CI})$} & \multirow{2}{*}{$\begin{array}{l}\mathrm{p} \text { - } \\
\text { value }\end{array}$} \\
\hline & & Yes (\%) & No (\%) & & \\
\hline \multirow[t]{4}{*}{ Educational status } & Illiterate & $43(41.3)$ & $61(58.7)$ & $.247(.106-.575)^{*}$ & \multirow[t]{4}{*}{.001} \\
\hline & Read \& write & $7(36.8)$ & $12(63.2)$ & $.298(.090-.987)$ & \\
\hline & Primary/HS & $14(15.9)$ & $74(84.1)$ & $.919(.358-2.361)$ & \\
\hline & College & $8(14.8)$ & $46(85.2)$ & 1 & \\
\hline \multirow[t]{2}{*}{ Address } & Urban & $25(15.4)$ & $137(84.6)$ & $\begin{array}{l}4.599(2.585- \\
8.184)\end{array}$ & \multirow[t]{2}{*}{.000} \\
\hline & Rural & $47(45.6)$ & $56(54.4)$ & 1 & \\
\hline \multirow{4}{*}{ Family size } & $1-2$ & $3(50.0)$ & $3(50.0)$ & $.889(.199-3.961)$ & \multirow[t]{4}{*}{.061} \\
\hline & $3-4$ & $22(22.0)$ & $78(78.0)$ & $1.842(.972-3.493)$ & \\
\hline & $5-6$ & $28(27.7)$ & $73(72.3)$ & $1.849(.903-3.787)$ & \\
\hline & $>6$ & $19(32.8)$ & $39(67.2)$ & 1 & \\
\hline \multirow[t]{4}{*}{ Occupation } & House wives & $49(31.4)$ & $107(68.6)$ & $.840(.423-1.666)$ & \multirow[t]{4}{*}{.067} \\
\hline & Daily laborer & $1(50.0)$ & $1(50.0)$ & $.385(.023-6.550)$ & \\
\hline & $\begin{array}{l}\text { Gov. } \\
\text { employee }\end{array}$ & $7(13.2)$ & $46(86.8)$ & $2.527(.936-6.825)$ & \\
\hline & Merchant & $15(27.8)$ & $39(72.2)$ & 1 & \\
\hline \multirow{3}{*}{$\begin{array}{l}\text { Average } \\
\text { income }\end{array}$} & $<1000 \mathrm{~EB}$ & $40(44.4)$ & $50(55.6)$ & $.260(.144-.470)$ & \multirow[t]{3}{*}{.000} \\
\hline & 1001-2000EB & $6(25.0)$ & $18(75.0)$ & $.624(.226-1.723)$ & \\
\hline & $>2000 \mathrm{~EB}$ & $26(17.2)$ & $125(82.8)$ & 1 & \\
\hline
\end{tabular}

Table 5. Binary Logistic Regression Analysis for Medical and Obstetric Associated Factors 


\begin{tabular}{|c|c|c|c|c|c|}
\hline \multirow[t]{2}{*}{ Variable } & \multirow[t]{2}{*}{ Categories } & \multicolumn{2}{|r|}{ Anemic } & \multirow[t]{2}{*}{ COR $\quad(95 \% \mathrm{CI})$} & \multirow{2}{*}{$\begin{array}{l}\mathrm{p}- \\
\text { value }\end{array}$} \\
\hline & & Yes (\%) & No (\%) & & \\
\hline \multirow[t]{2}{*}{ Abortion history } & Yes & $21(43.8)$ & $27(56.2)$ & $.274(.141-.533)$ & .000 \\
\hline & No & $51(23.5)$ & $\begin{array}{l}166 \\
(76.5)\end{array}$ & 1 & \\
\hline \multirow[t]{2}{*}{$\begin{array}{l}\text { Contraceptive } \\
\text { before }\end{array}$} & Yes & 17(17.0) & $83(83.0)$ & $\begin{array}{l}2.827(1.531- \\
5.219)\end{array}$ & .001 \\
\hline & No & $55(33.3)$ & $\begin{array}{l}110 \\
(66.7)\end{array}$ & 1 & \\
\hline \multirow[t]{2}{*}{$\begin{array}{l}\text { Heavy } \\
\text { bleeding }\end{array}$} & Yes & $\begin{array}{l}28 \\
(43.8)\end{array}$ & $36(56.2)$ & $.239(.131-.437)^{*}$ & .000 \\
\hline & No & $\begin{array}{l}44 \\
(21.9)\end{array}$ & $\begin{array}{l}157 \\
(78.1)\end{array}$ & 1 & \\
\hline \multirow[t]{4}{*}{ Inter-pregnancy interval } & 0 & $30(11.3)$ & $89(33.6)$ & $.364(.119-1.117)$ & .002 \\
\hline & $1-3$ & $10(3.8)$ & $28(10.6)$ & $.152(.025-.940)$ & \\
\hline & $4-6$ & $\begin{array}{l}28 \\
(10.6)\end{array}$ & $48(18.1)$ & $.309(.087-1.092)$ & \\
\hline & $>6$ & $4(1.5)$ & $28(10.6)$ & $.167(.053-.524)$ & \\
\hline \multirow[t]{3}{*}{ Gestational age } & $\begin{array}{l}\text { First } \\
\text { trimester }\end{array}$ & $7(30.4)$ & $16(69.6)$ & $\begin{array}{l}1.317(.493- \\
3.521)\end{array}$ & .008 \\
\hline & Second trim & $\begin{array}{l}31 \\
(20.8)\end{array}$ & $\begin{array}{l}118 \\
(79.2)\end{array}$ & $\begin{array}{l}2.194(1.230- \\
3.912)\end{array}$ & \\
\hline & Third trime & $\begin{array}{l}34 \\
(35.6)\end{array}$ & $59(63.4)$ & 1 & \\
\hline \multirow[t]{3}{*}{ ANC visits } & First & $24(20.0)$ & $96(80.0)$ & $\begin{array}{l}2.054(1.008- \\
4.184)\end{array}$ & .045 \\
\hline & Second & $29(32.6)$ & $60(67.4)$ & $\begin{array}{l}1.062(.523- \\
2.159)\end{array}$ & \\
\hline & $\begin{array}{l}\text { Third } \\
\text { above }\end{array}$ & 19(33.9) & $37(66.1)$ & 1 & \\
\hline
\end{tabular}

Table 6. Binary Logistic Regression Analysis for Nutrition associated Factors \& Anemia 


\begin{tabular}{|c|c|c|c|c|c|c|}
\hline \multirow[t]{2}{*}{ Variable } & & \multirow[t]{2}{*}{ Categories } & \multicolumn{2}{|c|}{ Anemic } & \multirow[t]{2}{*}{ COR 95\% CI } & \multirow{2}{*}{$\begin{array}{l}\mathrm{P}- \\
\text { value }\end{array}$} \\
\hline & & & Yes (\%) & No (\%) & & \\
\hline \multirow{3}{*}{\multicolumn{2}{|c|}{ Meal frequency }} & Once/day & $9(36.0)$ & $16(64.0)$ & $.425(.172-1.047)$ & \multirow[t]{3}{*}{.001} \\
\hline & & Twice/day & $31(41.9)$ & $43(58.1)$ & $.331(.181-.605)$ & \\
\hline & & $\begin{array}{l}\text { Three\& } \\
\text { more }\end{array}$ & $32(19.3)$ & $134(80.7)$ & 1 & \\
\hline \multirow{4}{*}{\multicolumn{2}{|c|}{$\begin{array}{l}\text { Consuming } \\
\text { vegetable }\end{array}$}} & Everyday & $3(13.0)$ & $20(87.0)$ & $\begin{array}{l}8.485(1.995- \\
36.093)\end{array}$ & \multirow[t]{4}{*}{.004} \\
\hline & & $\begin{array}{l}\text { Once per } \\
\text { wk. }\end{array}$ & $33(22.8)$ & $112(77.2)$ & $\begin{array}{l}4.320(1.792- \\
10.414)\end{array}$ & \\
\hline & & $\begin{array}{l}\text { Once } \\
\text { month }\end{array}$ & $22(30.6)$ & $50(69.4)$ & $\begin{array}{l}2.893(1.135- \\
7.371)\end{array}$ & \\
\hline & & Not at all & $14(66.0)$ & $11(44.0)$ & 1 & \\
\hline \multirow{2}{*}{\multicolumn{2}{|c|}{ IFAS }} & Yes & $42(31.1)$ & $93(68.9)$ & $.664(.384-1.148)$ & \multirow[t]{2}{*}{.018} \\
\hline & & No & $30(76.9)$ & $100(23.1)$ & 1 & \\
\hline \multirow{2}{*}{\multicolumn{2}{|c|}{ Coffee/tea drinking }} & Yes & $66(42.0)$ & $91(58.0)$ & $.178(.089-.360)$ & \multirow[t]{2}{*}{.000} \\
\hline & & No & $6(5.6)$ & $102(94.4)$ & 1 & \\
\hline \multirow{3}{*}{\multicolumn{2}{|c|}{ MUAC }} & $<23 \mathrm{~cm}$ & $\begin{array}{l}10 \\
(52.6)\end{array}$ & $9(47.4)$ & $.175(.052-.587)$ & \multirow[t]{3}{*}{.017} \\
\hline & & $21-23 \mathrm{~cm}$ & $\begin{array}{l}55 \\
(27.1)\end{array}$ & $\begin{array}{l}148 \\
(72.9)\end{array}$ & $.523(.22 .-1.245)$ & \\
\hline & & $>23 \mathrm{~cm}$ & $7(16.3)$ & $36(83.7)$ & 1 & \\
\hline
\end{tabular}

In Multivariate regression analysis results, the pregnant women who were from urban dwellers (AOR, 95\% CI: .18(.05-.64)), abortion before current pregnancy (AOR, 95\% CI: 3.08(1.17-8.13)), coffee/tea drinking status immediately after meal (AOR, 95\% CI: 4.39(1.82-10.59)), excessive menstrual bleeding than usual before the current pregnancy (AOR, 95\% CI: 3.39(1.47-7.84)) and Mid-Upper Arm Circumference (AOR, 95\% CI: $6.27(1.15-14.30)$ ) were independent predictors of anemia by controlling other explanatory variables (Table 7).

Table 7. Multivariate Logistic Regression Analysis Associated Factors with Anemia among pregnant women attending ANC at Yabello general hospital 17 June-16 August 2019 


\begin{tabular}{|c|c|c|c|}
\hline Variables & Categories & $\begin{array}{l}\text { COR } \\
p<0.25\end{array}\left(\begin{array}{ll}95 \% & \text { CI }),\end{array}\right.$ & $\mathrm{AOR}(95 \% \mathrm{CI}) \mathrm{p}<.05$ \\
\hline \multirow[t]{2}{*}{ Address } & Urban & $4.599(2.585-8.184)$ & $.178(.050-.635) * .008$ \\
\hline & Rural & & 1 \\
\hline \multirow[t]{2}{*}{ Abortion } & Yes & $.274(.141-.533)$ & $\begin{array}{l}3.081(1.167- \\
8.131) * .023\end{array}$ \\
\hline & No & & 1 \\
\hline \multirow[t]{2}{*}{ Coffee drinking } & Yes & $.178(.089-.360)$ & $\begin{array}{l}4.394(1.823- \\
10.589) * .001\end{array}$ \\
\hline & No & & 1 \\
\hline \multirow[t]{2}{*}{$\begin{array}{l}\text { Excessive } \\
\text { bleeding }\end{array}$} & Yes & $.239(.131-.437)$ & $\begin{array}{l}3.394(1.469- \\
7.844) * .004\end{array}$ \\
\hline & No & & 1 \\
\hline \multirow[t]{3}{*}{ MUAC } & $<23 \mathrm{~cm}$ & $.175(.052-.587)$ & $\begin{array}{l}6.274(1.148- \\
14.298) * .034\end{array}$ \\
\hline & $21-23 \mathrm{~cm}$ & $.523(.22 .-1.245)$ & 1 \\
\hline & $>23 \mathrm{~cm}$ & $.123(.12 .-.245)$ & 1 \\
\hline
\end{tabular}

\section{Discussion:}

The aim of study was to know the magnitude of anemia and associated factorst among pregnant women. According to finding, the overall magnitude of anemia is $27.2 \%$, this is similar when compare with study conducted in the South Nations and Nationalities People of Ethiopia which showed $(27.6 \%)$ (36); however, the finding is higher than a study conducted in Adama Hospital Medical College (14.9\%) (41), North Shoa (10\%) (37), Gondar (16.6\%) (31), and was slightly higher compared to the national prevalence (23\%) (10) and study conducted in China (23.5\%) (8). Furthermore, the finding is lower than the study findings conducted in the Arba-Minch hospital (32.8\%), Neqemte hospital (52.0\%), Kenya (57.0\%), South Africa, (57.7\%), India (64.0\%) and Baghdad (67.0\%)-(46, 40, 30, 22, 17 and 13). The differences might be because of the geographical variations, sampling techniques, software used for data analysis, skill of research teams, information and recall biases. Based up on the classification degree of anemia by WHO among all anemic pregnant women about (63.9\%) were mild anemic, 33.3\% were moderately anemic and (2.8\%) were severely anemic. Our study findings also contradict with the study conducted in Egypt (92.2\% mildly anemic, 7.8\% moderately anemic and no severely anemic was reported). This variation might be resulted from different geographic location and advancement in quality of ANC and standardized living conditions (29).

Multiple logistic regression analysis reveals that, the odds of anemia among pregnant women who were urban dwellers (AOR 95\% Cl: .178(.050-.635) times less likely compared to the odds of anemia among pregnant women who were rural dwellers. Similar finding of the study was reported from Ambiya health 
center (33). This variation could be due to; difference in educational level (55.5\% from urban compared to $5.3 \%$ from rural; at least with one of educational categories), being informed about anemia and the possible risk factors (46.4\%) urban dweller compared to pregnant women from rural (18.1) and being supplemented with IFA during their ANC visit $61.1 \%$ and $389 \%$ pregnant women from urban and rural respectively.

Among pregnant women who encountered abortion before the current pregnancy, the probability being anemic was 3.081times higher than pregnant women who did not encounter abortion before the current pregnancy (AOR=95\% Cl: (1.167-8.131)). This could be resulted because abortion might expose women to loss more blood through prolonged hemorrhage and this condition leads to extra requirements of iron by the body (4). Study also showed that pregnant women who used to drink coffee/tea immediately after meal were (AOR=95\% Cl: 4.394(1.823-10.589) times more likely to be anemic compared to pregnant women who did not drink coffee/tea immediately after meal. The similar study conducted in Durame health center showed similar result (48). The possible reason could be some chemicals could inhibit nonheme iron absorption by Tannin acid in coffee/tea. According to findings pregnant women with heavy menstrual bleeding than usual before the current pregnancy were (AOR=95\% Cl: 3.394(1.469-7.844)) times more affected by anemia compare to pregnant women with normal menstrual bleeding; this finding is similar with the study in Mizan Tepi (4). Because of excessive loss of blood through menstrual bleeding, the pregnant women might encounter anemia due to the expansion of the blood plasma volume. The odds of anemia among pregnant women with mid-upper arm circumference less than $23 \mathrm{~cm}$ were (AOR=95\% Cl: 6.274(1.148-14.298) which is lower than odds of similar study conducted in Kenya, Nairobi (30). In this study the findings show that, age, meal frequency per day, gravidity, not consuming green leafy vegetables, not consuming fruits after meal, history of malaria before the current pregnancy, not using Iron-Folic-Acid (IFA) supplementation and educational level, are not significantly associated with maternal anemia, but they were significantly associated with maternal anemia by other similar studies findings $(5,6,17,19,28$ \& 38-39)

\section{Conclusions:}

The research is intent to assess current status of anemia among pregnant women. Anemia prevalence in study area among pregnant women was moderate as compare with similar study conducted elsewhere. A multitude of external factors can affect the amount of iron available for absorption as either inhibitors or promoters. In this study malnutrition, abortion, excessive bleeding and nutrition interaction with other inhibitors like coca cola, tea and coffee drinking immediately after meals were independent predictors for anemia development. Therefore, health education should be given by health professional to enhance knowledge of pregnant on adequate dietary intake and Iron-folic combination supplement.

\section{Abbreviations}

ANC: Antenatal Care; CSA: Central Statistical Agency; EDHS: Ethiopian Demography and Health Survey; HGB: Hemoglobin; HSDP: Health Sector Development Program; IDA: Iron Deficiency Anemia;IFAS: Iron 
and Folic Acid Supplementation;

ITN: Insecticide Treated bed Net;MUAC: Mid-Upper Arm Circumference; RBCs: Red Blood Cells; SPSS: Statistical Package for Social Science;SSA: Sub-Saharan Africa

\section{Declarations}

\section{Ethics approval and consent to participate:}

Ethical clearance was obtained from the Institutional Ethical Review Board of Adama Hospital Medical College and subsequent permissions were obtained from line departments and respective health institutions. Data were collected through structured questionnaire after translated into local language from pregnant women attending antenatal care follow up at different private and government higher clinics and Hospital. Verbal informed consent was obtained from participants who visit the facilities during the data collection period. In the informed consent form significant information such as aim of the study, confidentiality of the information and participant's right to drop and withdraw from study have been explained.

Consent for publication: As there are no identifiable details on individual participants reported in the manuscript, consent to publish is not required.

Availability of data and materials: The datasets examined during the current study are available from the both authors corresponding and second authors on sensible inquiry.

Competing interests: As the authors we did not have any competing interest.

Funding: Adama Hospital Medical College delivered us financial support for the data collection and related cost. No other fund was obtained for the current

Authors' contributions: We are proposed and ran study design, data collection, performed data analysis and recruited the manuscript. Third persons had been analytically revised and finalized entire document with imperative contributions. Finally, we both authors have read and approved the final version of the manuscript.

Authors' information: Dr Godana Arero ( MPH in General Public Health, PhD in Nutritional Science) and working at Adama Hospital Medical College as Assistant Professor of Nutrition, researcher, consultant and Head Public Health Department. Mr. Kinde Asssefa (BSC in Laboratory Technology) working at Yabello General Hospital, Department of Laboratory as a expert.

Acknowledgements: We thank Adama Hospital Medical College for funding the study and for the ethical approval. We are also thankful to other line departments and individuals who played important roles in 
availing necessary information without which this work wouldn't have been completed. Last, but not least, we also appreciate the contributions of all supervisors, data collectors and study participants for their respective contribution without which the work would not have been appreciated.

\section{References}

1. Centers for Disease Control (CDC) CDC criteria for anemia in children and childbearing-aged women. MMWR Morb Mortal Wkly Rep. 1989;38:400-4. - PubMed

2. Davas I, Marangoz D, Varolan A, Akyol A, Baksu B. Gebelikte değişik seviyelerdeki anemilerde demir alınımının maternal, doğum ve perinatal sonuçlara etkileri. J Turk Soc Obstet Gynecol. 2008;5:17481.

3. World Health Organization. Worldwide prevalence of anemia 1993-2005. WHO Global Database on Anemia. Geneva: World Health Organization. 2008

4. World Health Organization. Iron deficiency anemia assessment prevention and control: a guide for programme managers. Geneva: World Health Organization. 2001;132.

5. Ren A, Wang J, Ye RW, Li S, Liu JM, Li Z. Low first-trimester hemoglobin and low birth weight, preterm birth and small for gestational age newborns. Int J Gynaecol Obstet. 2007;98:124-8. - PubMed

6. Sekhavat L, Davar R, Hosseinidezoki S. Relationship between maternal hemobglobin concentration and neonatal birth weight. Hematology. 2011;16:373-6. - PubMed

7. Segel GB, Hirsh MG, Feig SA. Managing anemia in pediatric office practice: Part 1. Pediatr Rev. 2002;23:75-84. - PubMed

8. Wu AC, Lesperance L, Bernstein H. Screening for iron deficiency. Pediatr Rev. 2002;23:171-8. PubMed

9.World Health Organization WHO. Maternal Health and Safe Motherhood Programme. Maternity care: what is needed for safer motherhood in the community? Safe Mother. 1992:6-8. - PubMed

10. Tolentino K. and Friedman JF. An Update on Anemia in Less Developed Countries. Am J Trop Med Hyg. 2007, 77 (1):4.

11. Allen LH. Pregnancy and iron deficiency: unresolved issues. Nutr Rev. 1997;55:91-101. - PubMed

12. Bestepe G, Bilgin N. Afyon ili 2 ve 4 No'lu sağhk ocaklarındaki gebelerde anemi prevalansı ve anemiyi etkileyen bazı faktörlerin incelenmesi. Sağlık ve Toplum. 2002;12:43-53.

13. Pirinçci E, Açık Y, Bostancı M, Eren S, Beritanlı H. Elazığ il merkezinde yaşayan gebelerde anemi prevalansı. F.Ü.Sağ.Bil.Tıp Derg. 2001;15:449-54.

14. Karaoglu L, Pehlivan E, Egri M, Deprem C, Gunes G, Genc MF, et al. The prevalence of nutritional anemia in pregnancy in an east Anatolian province, Turkey. BMC Public Health. 2010;10:329. - PMC PubMed 
15. Mekonin F., Abere Y., and Timerga G. The socio-economic determinants of anemia in pregnancy in north Shoa zone, Ethiopia. Ethiopian Medical Journal. 2018, 13(8):28.https://doi.org/10.1371/journal.pone.0202734

16. Scholl TO. Iron status during pregnancy: setting the stage for mother and infant. Am J Clin Nutr. 2005;81:1218-22. - PubMed

17. Malhotra M, Sharma JB, Batra S, Sharma S, Murthy NS, Arora R. Maternal and perinatal outcome in varying degrees of anemia. Int J Gynecol Obstet. 2002;79:93-100. - PubMed

18. WHO/CDC. Worldwide Prevalence of Anemia 1993-2005. WHO Global Database on Anemia. Geneva, Switzerland: WHO Press; 2008.

19. Balarajan Y, Ramakrishnan U, Ozaltin E, Shankar AH, Subramanian SV. Anaemia in low-income and middle-income countries. Lancet. 2011;378(9809):2123-2135. - PubMed

20. Salhan S, Tripathi V, Singh R, Gaikwad HS. Evaluation of hematological parameters in partial exchange and packed cell transfusion in treatment of severe anemia in pregnancy. Anemia. 2012;2012:608658. - PMC- PubMed

21. Akhtar M, Hassan I. Severe anemia during late pregnancy. Case Report Obstet Gynecol. 2012;2012:3.

22. Baker SJ, DeMaeyer EM. Nutritional anemia: its understanding and control with special reference to the work of the world health organization. Am J Clin Nutr. 1979;32(2):368-417. - PubMed

23. World Health Organization . Prevention and management of severe anaemia in pregnancy: report of a technical working group, Geneva, 20-22 May 1991. 1993.

24. Stevens GA, Finucane MM, De-Regil LM, Paciorek CJ, Flaxman SR, Branca F, Peña-Rosas JP, Bhutta ZA, Ezzati M, Group NIMS Global, regional, and national trends in haemoglobin concentration and prevalence of total and severe anaemia in children and pregnant and non-pregnant women for 1995-2011: a systematic analysis of population-representative data. Lancet Glob Health. 2013;1(1):e16-e25. doi: 10.1016/S2214-109X(13)70001-9. - DOI- PMC- PubMed

25. Ghana Statistical Service, Ghana Health Service, Measure DHS . Ghana: demographic and health survey 2014. 2015.

26. World Health Organization. Global nutrition targets 2025: anaemia policy brief. 2014.

27. Brabin BJ, Hakimi M, Pelletier D. An analysis of anemia and pregnancy-related maternal mortality. J Nutr. 2001;131(2):604S-615S. doi: 10.1093/jn/131.2.604S. - DOI- PubMed

28. World Health Organization. The Global Prevalence of Anaemia in 2011. Geneva: World Health Organisation; 2015.

29. Balarajan Y, Ramakrishnan U, Özaltin E, Shankar AH, Subramanian SV. Anaemia in low-income and middle-income countries. Lancet. 2011;378(9809):2123-2135. - PubMed

30. Gebre A, Mulugeta A. Prevalence of anemia and associated factors among pregnant women in north western zone of Tigray, Northern Ethiopia: a cross-sectional study. Journal of Nutrition and Metabolism. 2015;2015:165430. - PMC- PubMed 
31. Obai G, Odongo P, Wanyama R. Prevalence of anaemia and associated risk factors among pregnant women attending antenatal care in Gulu and Hoima Regional Hospitals in Uganda: a cross sectional study. BMC Pregnancy and Childbirth. 2016 Apr 11;16:76. - PMC- PubMed

32. Dattijo LM, Daru PH, Umar NI. Anaemia in Pregnancy: prevalence and associated factors in Azare, North-East Nigeria. International Journal of Tropical Disease \& Health. 2016;11(1):1-9.

33. Chathuranga G, Balasuriya T, Perera R. Anaemia among female undergraduates residing in the hostels of University of Sri Jayewardenepura, Sri Lanka. Anemia. 2014 - PMC- PubMed

34. Goonewardene M, Shehata M, Hamad A. Anaemia in pregnancy. Best Pract Res Clin Obstet Gynaecol. 2012;26(Suppl 1):3-24. doi: 10.1016/j.bpobgyn.2011.10.010. - DOI- PubMed

35. Rush D. Nutrition and maternal mortality in the developing world. Am J Clin Nutr. 2000;72(Suppl 1):212-240. - PubMed

36. Khan KS, Wojdyla D, Say L, Gülmezoglu AM, Van Look PF. WHO analysis of causes of maternal death: a systematic review. Lancet. 2006 - PubMed

37. Asre F. Magnitude of anemia and its associated factors among pregnant women receiving antenatal care at Ambiya health center northwest Ethiopia. Journal of blood medicine. 2017, 3(8):35-1.

38. McLean E, Cogswell M, Egli I, Wojdyla D, de Benoist B. Worldwide prevalence of anaemia, WHO vitamin and mineral nutrition information system, 1993-2005. Public Health Nutr. 2009;12(4):444454. 10.1017/S1368980008002401 - DOI- PubMed

39. Ozturk M., Ozgurtas T., Yemen M., and Firatligil F. Anemia magnitude at the time of pregnancy detection Turkey. Turkish Journal of Obstetrics and Gynecology. 2017, 14(3): 176.

40. Erhabor O, Isaac IZ, Isah A, Udomah FP. Iron deficiency anaemia among antenatal women in Sokoto, Nigeria. Br J Med Health Sci. 2013;1(4):47-57.

41. Mihiretie H., Fufa M., Mitiku A., et al. Magnitude of anemia and associated factors among pregnant women attend antenatal care in, Nekemte, Ethiopia. J Med Microb Diagn. 2015, 4(3):4.

42. Msuya SE, Hussein TH, Uriyo J, Sam NE, Stray-Pedersen B. Anaemia among pregnant women in northern Tanzania: Prevalence, risk factors and effect on perinatal outcomes. Tanzan $\mathrm{J}$ Health Res. 2011;13(1):33-39. 10.4314/thrb.v13i1.60881 - DOI- PubMed

43. Melku M, Addis Z, Alem M, Enawgaw B. Prevalence and predictors of maternal anemia during pregnancy in Gondar, Northwest Ethiopia: An institutional based cross-sectional study. Anemia. 2014;2014:108593. 10.1155/2014/108593 - DOI- PMC- PubMed

44 Bodeau-Livinec F, Briand V, Berger J, et al. Maternal anemia in Benin: Prevalence, risk factors, and association with low birth weight. Am J Trop Med Hyg. 2011;85(3):414-420. 10.4269/ajtmh.2011.100599 - DOI - PMC - PubMed

45. Bimrew L., Ayele A., and Mossie A. Anemia and associated factors among pregnant women attending antenatal care clinic in Welayita Sodo Ethiopia. Ethiopian journal of health science. 2015, 25(2):155. 
46. Grewal A. Anaemia and pregnancy: Anaesthetic implications. Indian J Anaesth. 2010;54(5):380-386. doi: 10.4103/0019-5049.71026. - DOI- PMC- PubMed

47. Stevens GA, Finucane MM, De-Regil LM, Paciorek CJ, Flaxman SR, Branca F, et al. Global, regional, and national trends in haemoglobin concentration and prevalence of total and severe anaemia in children and pregnant and non-pregnant women for 1995-2011: a systematic analysis of populationrepresentative data. Lancet Glob Health. 2013;1(1):e16-e25. doi: 10.1016/S2214-109X(13)70001-9.

- DOI- PMC- PubMed

48. WHO . Haemoglobin concentrations for the diagnosis of anaemia and assessment of severity. Edited by World Health Organization. 2011.

49.World Health Organization. Worldwide prevalence of anaemia 1993-2005: WHO global database on anaemia. Edited by Bruno de Benoist, Erin McLean, Ines Egli and Mary Cogswell. 2008.

\section{Figures}

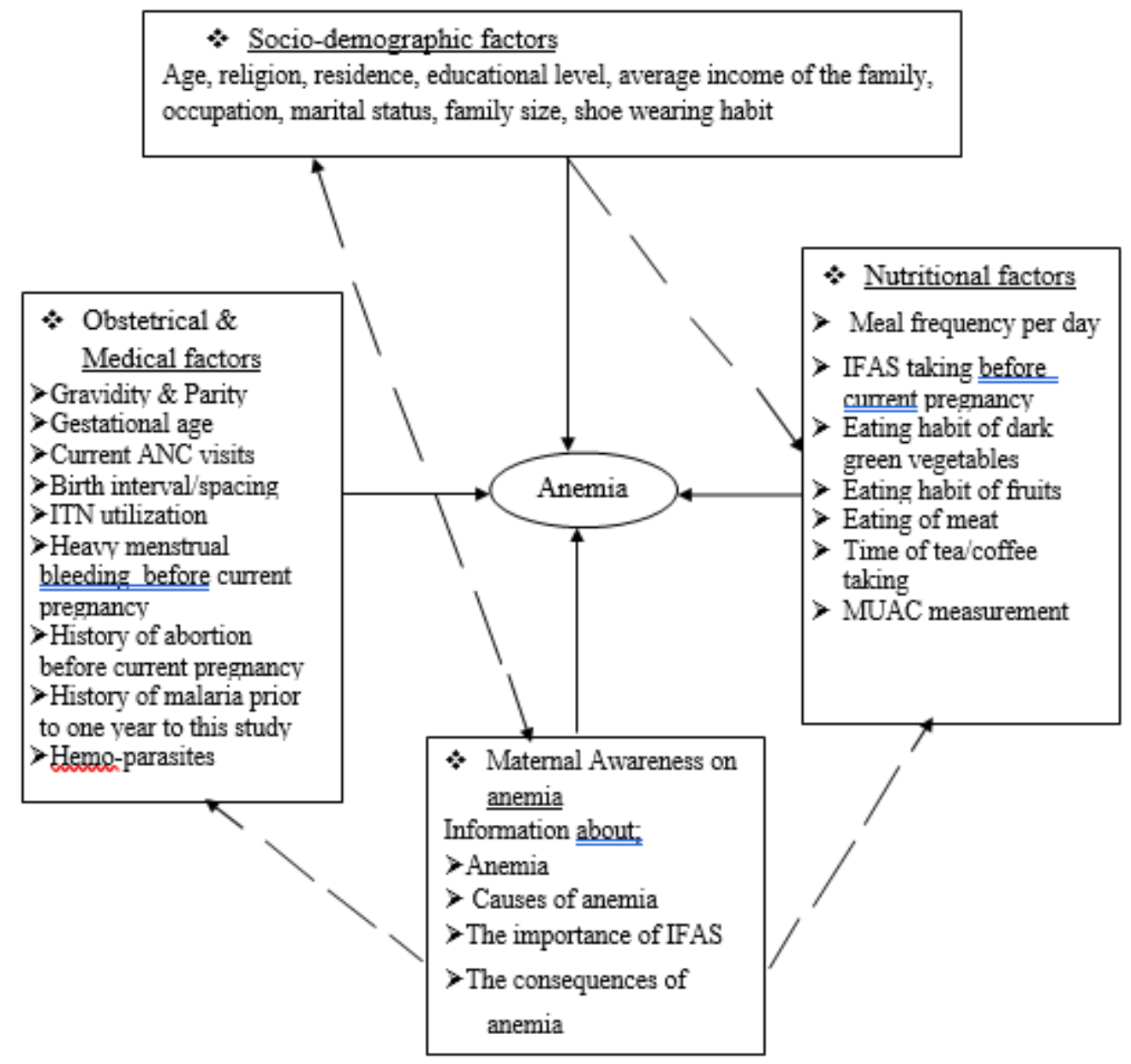

\section{Figure 1}

The Conceptual Frame work adapted from Abie A. 2018 


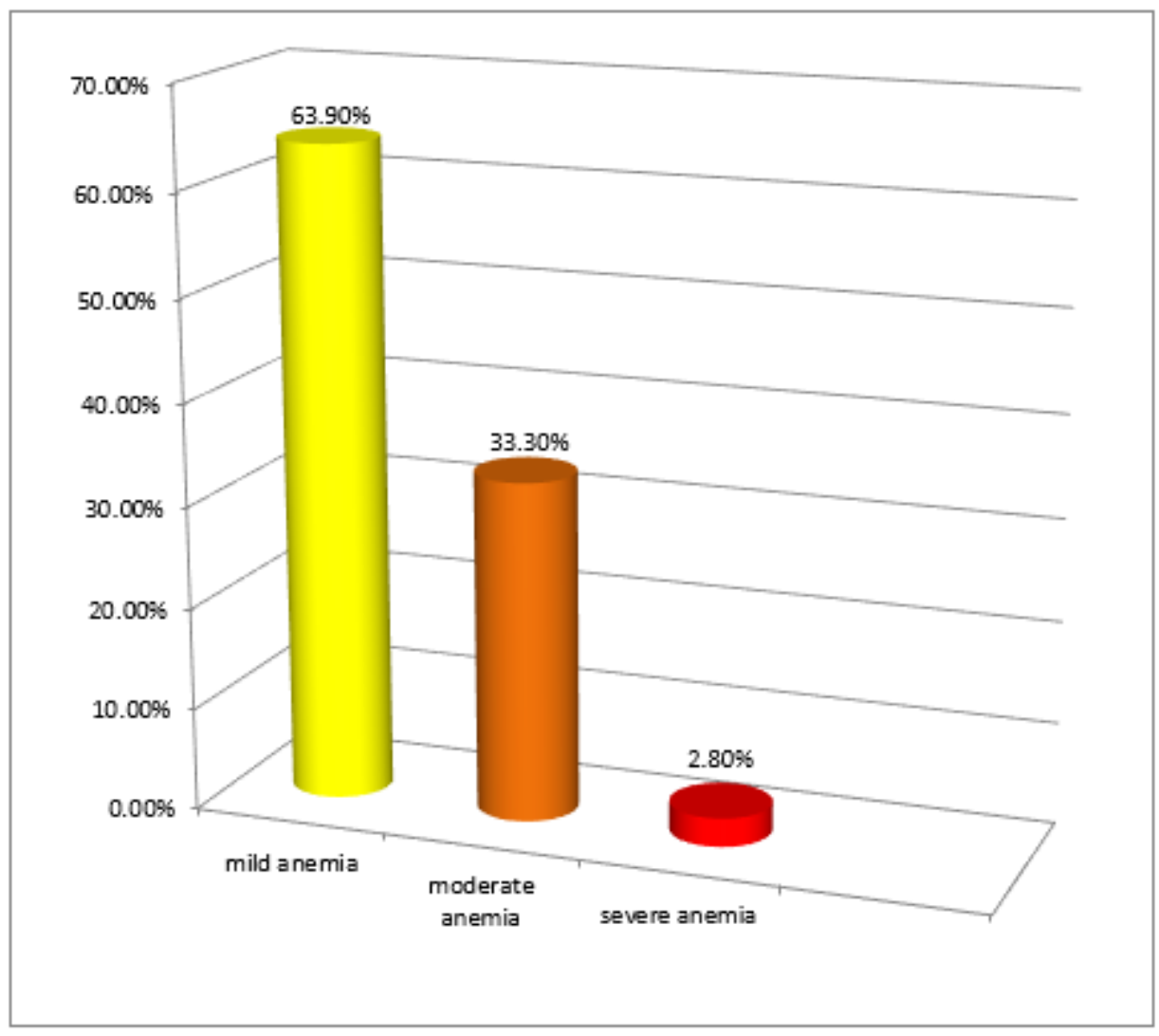

Figure 2

Percentage of anemia by severity among anemic pregnant women $(n=72)$

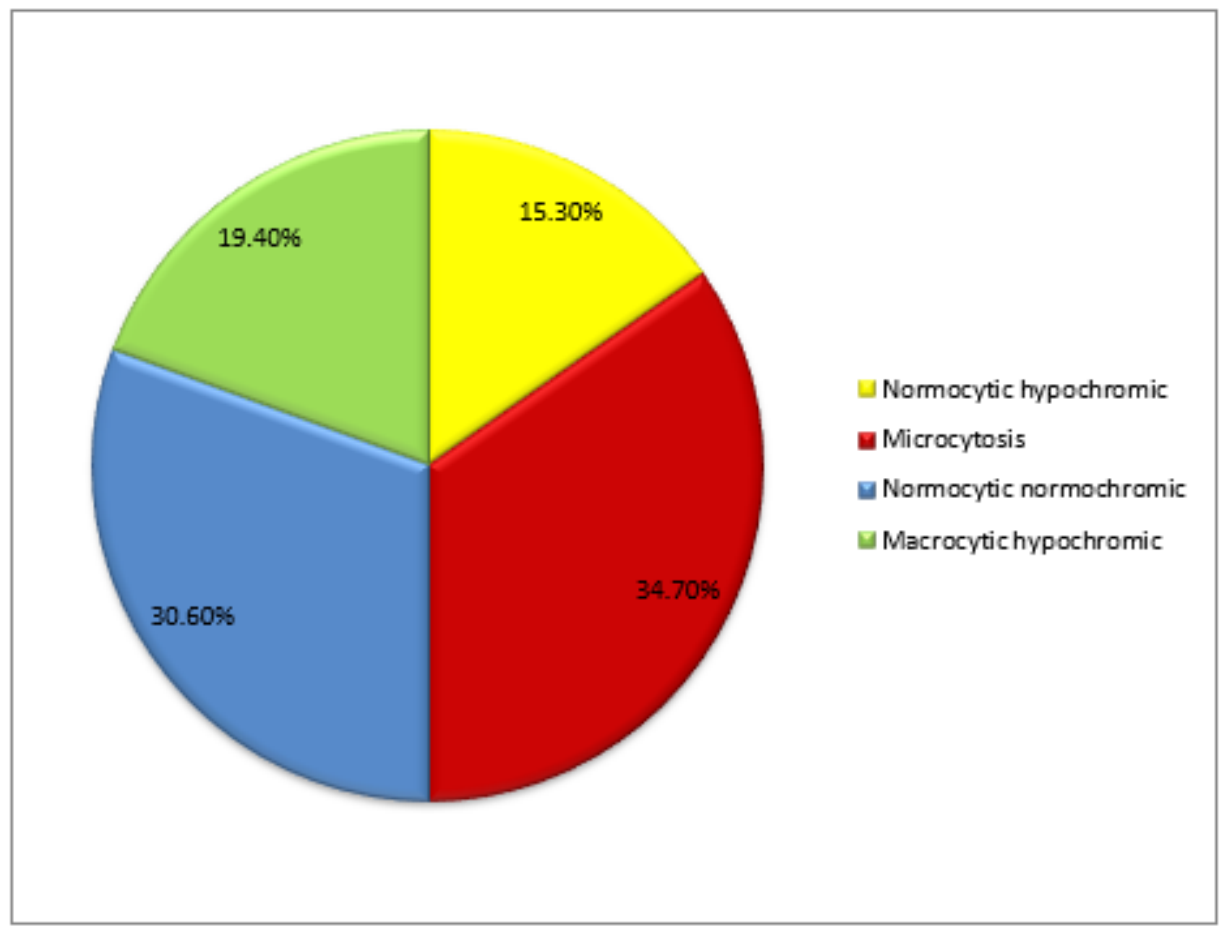

Figure 3

Red blood cells morphologic distribution among anemic pregnant women $(n=72)$ 